\title{
Prolonged Screen Viewing Times and Sociodemographic Factors among Pregnant Women: A Cross-Sectional Survey in China
}

\author{
Xianglong $X u^{1,2,3,+(D)}$, Dengyuan Liu ${ }^{1,2,3,+}$, Yunshuang Rao ${ }^{1,2,3}$, Huan Zeng 1,2,3, \\ Fan Zhang ${ }^{1,2,3}$, Lu Wang ${ }^{1,2,3}$, Yaojie Xie ${ }^{4}$, Manoj Sharma ${ }^{5}$ and Yong Zhao ${ }^{1,2,3, *}$ \\ 1 School of Public Health and Management, Chongqing Medical University, Chongqing 400016, China; \\ xianglong1989@126.com (X.X.); dengyuanliu@foxmail.com (D.L.); rys0606@163.com (Y.R.); \\ zenghuan586@aliyun.com (H.Z.); ava11@126.com (F.Z.); w1532916490@163.com (L.W.) \\ 2 Research Center for Medicine and Social Development, Chongqing Medical University, \\ Chongqing 400016, China \\ 3 Collaborative Innovation Center of Social Risks Governance in Health, Chongqing Medical University, \\ Chongqing 400016, China \\ 4 School of Nursing, The Hong Kong Polytechnic University, Hong Kong 999077, China; yaojie.xie@gmail.com \\ 5 Department of Behavioral and Environmental Health, Jackson State University, Jackson, MS 39213, USA; \\ manoj.sharma@jsums.edu.cn \\ * Correspondence: zhaoyong@cqmu.edu.cn; Tel.: +86-138-8346-0842; Fax: +86-023-6848-5031 \\ + These authors contributed equally to this work.
}

Received: 2 January 2018; Accepted: 19 February 2018; Published: 27 February 2018

\begin{abstract}
Objectives: This study aimed to assess the prevalence of prolonged television, computer, and mobile phone viewing times and examined related sociodemographic factors among Chinese pregnant women. Methods: In this study, a cross-sectional survey was implemented among 2400 Chinese pregnant women in 16 hospitals of 5 provinces from June to August in 2015, and the response rate of $97.76 \%$. We excluded women with serious complications and cognitive disorders. The women were asked about their television, computer, and mobile phone viewing during pregnancy. Prolonged television watching or computer viewing was defined as spending more than two hours on television or computer viewing per day. Prolonged mobile phone viewing was watching more than one hour on mobile phone per day. Results: Among 2345 pregnant women, about 25.1\% reported prolonged television viewing, $20.6 \%$ reported prolonged computer viewing, and $62.6 \%$ reported prolonged mobile phone viewing. Pregnant women with long mobile phone viewing times were likely have long TV (Estimate $=0.080$, Standard Error $(S E)=0.016, p<0.001$ ) and computer viewing times (Estimate $=0.053, S E=0.022, p=0.015$ ). Pregnant women with long TV (Estimate $=0.134$, $S E=0.027, p<0.001)$ and long computer viewing times (Estimate $=0.049, S E=0.020, p=0.015)$ were likely have long mobile phone viewing times. Pregnant women with long TV viewing times were less likely to have long computer viewing times (Estimate $=-0.032, S E=0.015, p=0.035$ ), and pregnant women with long computer viewing times were less likely have long TV viewing times (Estimate $=-0.059, S E=0.028, p=0.035$ ). Pregnant women in their second pregnancy had lower prolonged computer viewing times than those in their first pregnancy (Odds Ratio (OR) 0.56, 95\% Confidence Interval (CI) 0.42-0.74). Pregnant women in their second pregnancy were more likely have longer prolonged mobile phone viewing times than those in their first pregnancy (OR 1.25, 95\% CI 1.01-1.55). Conclusions: The high prevalence rate of prolonged TV, computer, and mobile phone viewing times was common for pregnant women in their first and second pregnancy. This study preliminarily explored the relationship between sociodemographic factors and prolonged screen time to provide some indication for future interventions related to decreasing screen-viewing times during pregnancy in China.
\end{abstract}


Keywords: screen time; TV viewing; computer viewing; mobile phone viewing; pregnant women; China

\section{Introduction}

Prolonged screen viewing times are common globally. Prolonged screen viewing times have been documented as prevalent sedentary behaviors associated with an increased risk of adverse pregnancy outcomes and chronic diseases. The association is found between prolonged mobile phone viewing and low birth weight, high infant emergency transfer rate [1], and shorter pregnancy duration [2]. Prolonged screen viewing times are also adversely associated with physical well-being, mental well-being, and vitality [3]. Obesity [4,5], developing diabetes [6], hypertension [7], and several musculoskeletal health complaints [8] are also associated with long screen viewing times. In addition, prolonged mobile phone usage is associated with delayed bedtime, short sleeping time [9,10], brain tumors, and parotid gland tumor [11]. Television viewing times have an inverse association with adjusted telomere length among Southwest Chinese adults [12].

According to the 2002 Chinese residents of nutrition and health survey, about $92.1 \%$ of Chinese residents watch TV, with an average time of $2.1 \mathrm{~h}$ every day [13], and $60.6 \%$ of these Chinese residents watch TV for over $2 \mathrm{~h}$ daily [14]. Approximately 59\% of American adults aged 20 years and older watch over $2 \mathrm{~h}$ of TV daily [15]. Computer usage has become one of the most prevalent factors affecting screen viewing times in China [16]. The average computer exposure time of a Chinese female is $2.9 \mathrm{~h} /$ day during 2011-2012 [14]. Mobile phone subscribers will increase from 4.8 billion in 2016 to 5.7 billion in 2020 globally [17]. In 2016, more than half of Chinese people used mobile phone more than $2 \mathrm{~h}$ per day [18].

Sociodemographic factors, such as age [14,19], gender [10,14,20,21], income level and labor intensity [22], educational level [19,23], residence [24], transportation options [25], business setting [26,27], and consumption level and frequency of use of the entertainment functions [10] are associated with prolonged screen viewing times. Previous studies have explored some sociodemographic factors related to prolonged screen viewing times, primarily among adults and children. Moreover, with the issuance of the Chinese new policy "two-child policy" in 2015, the number of pregnant women will decrease. Thus, the present study incorporates related factors, including parity and trimester, apart from sociodemographic factors.

To our knowledge, the prevalence of prolonged screen viewing times among pregnant women is unclear, and few studies have focused on the sociodemographic factors of prolonged screen viewing times among pregnant women, especially in China. This study provides an improved implication and understanding of the policies in Healthy China 2030 and National Nutrition Program by optimizing a healthy lifestyle among pregnant women. This study aims to assess prolonged TV viewing times, computer usage times, mobile phone viewing times, and their influencing factors (socioeconomic characteristics factors, trimester, and parity) among Chinese pregnant women to solve the following three objectives:

- To determine the prevalence rate of prolonged TV viewing, computer usage, and mobile phone viewing times among pregnant women in China;

- To indicate the association among prolonged TV viewing, computer usage, and mobile phone viewings time;

- To identify the factors affecting prolonged TV viewing, computer usage, and mobile phone viewing times among Chinese pregnant women. 


\section{Materials and Methods}

\subsection{Study Design and Participants}

The design and methods, sampling framework, survey administration, and pilot study, as well as the population and sample questionnaire development process, of the current study have been reported previously [28,29]. In brief, a total of 2400 pregnant women were surveyed by trained medical students, with a response rate of $97.76 \%$. Women with serious complications and cognitive disorders were excluded. All pregnant women visiting 16 hospitals in Chongqing, Chengdu, Zunyi, Liaocheng, and Tianjin were invited to participate in the survey from June to August in 2015. Chongqing, Chengdu, and Zunyi are in south China, whereas Liaocheng and Tianjin are in north China. The study protocol was ratified by the Ethics Committee of Chongqing Medical University (record number 2015008). All subjects provided their informed consent for inclusion before their participation.

\subsection{Questionnaire}

\subsubsection{Sociodemographic Variables}

Sociodemographic data included residence (Urban/Rural) and per capita monthly income of the family (low income (<¥4500)/medium income families ( $¥ 4500-¥ 9000) /$ high income families (>¥9000)), ( 1 USD $=¥ 6.79$ in June 2017)). Other categories included age ( $18-25$ years / $26-35$ years/36-45 years), occupation (rural migrant workers/urban and rural unemployed, unemployed/industrial workers of non-agricultural registered permanent residence/individual business/business services staff/civil servants/senior and middle-level managers in large and medium enterprises/private entrepreneur/professionals/clerks/students/others), hospital capacity/quality rank (high, medium, and low), nationality (Han nationality/minority), without sibling (yes/no), marital status (unmarried/married/remarried/divorced/widowed). Pregnancy was divided into three trimesters (first, second, and third trimester). Education level was categorized as low (junior, middle school, or below), medium (senior high school, vocational or technical secondary school), or high (university). Based on the Chinese hospital ranking system, the hospital capacity/quality rank was recorded as high, medium, or low [30].

In the multivariable analysis on watching over two hours of TV daily, computer usage times of more than two hours per day, and mobile phone viewing times of more than one hour per day, occupational category was categorized as manual (rural migrant workers/industrial workers of non-agricultural registered permanent residence/business services staff), non-manual (individual business/civil servants/senior manager and middle-level manager in large and medium enterprise/private entrepreneur/professionals/clerk/students), or unemployed.

\subsubsection{Outcome Variables}

Participants were asked about their total TV viewing, computer usage (including computer time at work), and mobile phone viewing times daily during pregnancy per day. Compared with adults without health conditions, a higher percentage of adults with health conditions watched over two hours of TV and computer per day [31]. Exposure to mobile phone usage for more than $1 \mathrm{~h}$ daily and parotid tumor was observed to be associated [32]. Prolonged watching times were defined as when a woman watched over two hours on TV or computer and watched over one hour on mobile phone per day. Therefore, participants were divided into three categories: TV viewing times (more than two hours per day and less than two hours per day), computer usage times (more than two hours per day and less than two hours per day), and mobile phone viewing times (more than one hour per day and less than one hour per day). 


\subsection{Statistical Analyses}

Participants' characteristics were summarized using either the means and standard deviations or the frequencies and percentages, which were presented using descriptive analysis (means, standard deviations, and percentages). Generalized linear model was utilized to probe factors that affected TV, computer, and mobile phone viewing times. Factors that extended TV, computer, and mobile phone viewing times were considered in the generalized linear model among pregnant women: TV viewing times, mobile phone usage times, computer usage times, parity, hospital level, occupational category, nationality, being a single-child, husband being a single-child, marital status, educational level, residence, age, trimester, and per capita monthly income of the family.

We included mobile phone usage times, hospital level, occupational category, without siblings, per capita monthly income of the family, and "prolonged TV viewing times" as dependent variables in the multivariate logistic regression model with backward elimination. Multivariate model was statistically significant in the model coefficient test $(p<0.05)$ and reached a good fit in Hosmer and Lemeshow test $(p=0.78)$. We included mobile phone viewing times, age, hospital level, occupational category, without siblings, education level, residence, per capita monthly income of the family, and trimester of pregnancy with "prolonged computer usage times" as the dependent variable in the multivariate logistic regression model with backward elimination; multivariate model was statistically significant in the model coefficient test $(p<0.05)$ and reached a good fit in Hosmer and Lemeshow test $(p=0.25)$. We included TV viewing times, computer usage times, age, occupational category, marital status, education level, and trimesters of pregnancy with "prolonged mobile phone usage times" as dependent variables in the multivariate logistic regression model with backward elimination. Multivariate model was statistically significant in the model coefficient test $(p<0.05)$ and reached a good fit in Hosmer and Lemeshow test $(p=0.54)$. All statistics were performed using 2-sided test, and statistical significance was considered at $p<0.05$. All data analyses were performed using statistical software (SAS version 9.1.3; SAS Institute, Cary, NC, USA).

\section{Results}

\subsection{Characteristics of Pregnant Women}

Of the 2345 participants (28.12 \pm 4.1 years), 1755 (74.8\%) were in their first pregnancy, and $590(25.2 \%)$ are in their second pregnancy. About $96 \%$ of pregnant women were of Han nationality. (Please see Table 1).

Table 1. Characteristics of the study participants in Chongqing, China, $2015(n, \%)$.

\begin{tabular}{ccc}
\hline Variable & Frequency & Percentage \\
\hline Age & & \\
18-25 years old & 624 & 26.6 \\
26-35 years old & 1595 & 68.0 \\
36-45 years old & 126 & 5.4 \\
\hline Parity & & \\
Pregnant women in first pregnancy & 1755 & 74.8 \\
Pregnant women in second pregnancy & 590 & 25.2 \\
\hline Hospital level & & \\
Level 3A hospital & 1824 & 77.8 \\
Level 2 A hospitals & 311 & 13.2 \\
Level 2B hospitals and below & 210 & 9.0 \\
\hline Nationality & & \\
Han nationality & 2252 & 96.0 \\
Non-Han nationality & 93 & 4.0 \\
\hline
\end{tabular}


Table 1. Cont.

\begin{tabular}{|c|c|c|}
\hline Variable & Frequency & Percentage \\
\hline \multicolumn{3}{|l|}{ Without siblings } \\
\hline Yes & 1046 & 44.6 \\
\hline No & 1299 & 55.4 \\
\hline \multicolumn{3}{|l|}{ Husband without siblings } \\
\hline Yes & 1173 & 50.0 \\
\hline No & 1172 & 50.0 \\
\hline \multicolumn{3}{|l|}{ Marital status } \\
\hline Unmarried & 49 & 2.1 \\
\hline Primary marriage & 2205 & 94.0 \\
\hline Remarried & 70 & 3.0 \\
\hline Divorced or Widowed & 21 & 0.9 \\
\hline \multicolumn{3}{|l|}{ Education level } \\
\hline Basic education & 402 & 17.1 \\
\hline Secondary education & 354 & 15.1 \\
\hline Higher education & 1589 & 67.8 \\
\hline \multicolumn{3}{|l|}{ Residence } \\
\hline Urban & 1880 & 80.2 \\
\hline Rural & 465 & 19.8 \\
\hline \multicolumn{3}{|l|}{ The per capital monthly income of the family } \\
\hline$<¥ 4500$ & 611 & 26.0 \\
\hline$¥ 4500$ and $¥ 9000$ & 989 & 42.2 \\
\hline$>¥ 9000$ & 745 & 31.8 \\
\hline \multicolumn{3}{|l|}{ Occupation } \\
\hline Rural migrant workers & 118 & 5.0 \\
\hline Urban and rural unemployed & 553 & 23.6 \\
\hline $\begin{array}{c}\text { Industrial workers of Non-agricultural registered permanent } \\
\text { residence }\end{array}$ & 50 & 2.1 \\
\hline Individual business & 199 & 8.5 \\
\hline Business services staff & 155 & 6.6 \\
\hline Civil servants & 398 & 17.0 \\
\hline $\begin{array}{c}\text { Senior managers and Middle-level managers in large and } \\
\text { medium enterprises }\end{array}$ & 96 & 4.1 \\
\hline Private entrepreneur & 87 & 3.7 \\
\hline Professionals & 244 & 10.4 \\
\hline Clerk & 139 & 5.9 \\
\hline Students & 15 & 0.7 \\
\hline Other & 291 & 12.4 \\
\hline \multicolumn{3}{|l|}{ Occupational category } \\
\hline Non-manual & 1178 & 50.2 \\
\hline Manual & 323 & 13.8 \\
\hline Unemployed & 553 & 23.6 \\
\hline Others & 291 & 12.4 \\
\hline \multicolumn{3}{|l|}{ Trimester of pregnancy } \\
\hline First trimester & 293 & 12.5 \\
\hline Second trimester & 701 & 29.9 \\
\hline Third trimester & 1351 & 57.6 \\
\hline
\end{tabular}

\subsection{Screen Times of Pregnant Women}

Daily TV viewing times of between two and four hours were reported by $12.5 \%$ of respondents. Among pregnant women, 9.4\% watched TV for 4-6 h and 3.1\% spent more than $6 \mathrm{~h}$ viewing TV daily.

Daily computer viewing times between two and four hours were $4.6 \%$. Among pregnant women, $6.4 \%$ watched computer for $4-6 \mathrm{~h}$ and $9.6 \%$ spent more than $6 \mathrm{~h}$ on computer viewing per day. 
Daily mobile phone usage times between one and two hours were $26.5 \%$. Among pregnant women, $14.3 \%$ viewed mobile phones for $2-4 \mathrm{~h}, 13.8 \%$ viewed mobile phones for $4-6 \mathrm{~h}$, and $7.9 \%$ spent more than $6 \mathrm{~h}$ on mobile viewing per day (see Table 2).

Table 2. Screen times of pregnant women in Chongqing, China $(n, \%)$.

\begin{tabular}{cccc}
\hline $\begin{array}{c}\text { Total Screen Time } \\
(\mathbf{5 . 9 0} \pm \mathbf{3 . 7 0} \mathbf{~})\end{array}$ & TV Viewing Times & Computer Viewing Times & Mobile Viewing Times \\
\hline Mean \pm SD & $1.80 \pm 1.68 \mathrm{~h}$ & $1.65 \pm 2.32 \mathrm{~h}$ & $2.45 \pm 2.18 \mathrm{~h}$ \\
$\leq 1$ & $1134(48.4)$ & $1572(67.0)$ & $878(37.4)$ \\
$(1,2]$ & $623(26.6)$ & $291(12.4)$ & $622(26.5)$ \\
$(2,3)$ & $14(0.6)$ & $5(0.2)$ & $14(0.6)$ \\
{$[3,4)$} & $280(11.9)$ & $102(4.4)$ & $322(13.7)$ \\
{$[4,5)$} & $137(5.8)$ & $83(3.5)$ & $203(8.7)$ \\
{$[5,6)$} & $84(3.6)$ & $68(2.9)$ & $120(5.1)$ \\
$\geq 6$ & $73(3.1)$ & $224(9.6)$ & $186(7.9)$ \\
\hline
\end{tabular}

\subsection{Prolonged TV Viewing, Computer Usage, and Mobile Phone Usage Times among Pregnant Women}

Prolonged TV viewing times were reported by 456 (26.0\%) and $132(22.4 \%)$ participants on their first and second pregnancies, respectively. A total of $466(24.8 \%)$ and $122(26.2 \%)$ participants form urban and rural areas, respectively, were reported to watch over two hours of TV per day (see Table 3).

Table 3. TV viewing, computer using and mobile phone usage times among pregnant women, China, $2015(n, \%)$.

\begin{tabular}{|c|c|c|c|c|c|c|}
\hline \multirow{2}{*}{ Variable } & \multicolumn{2}{|c|}{ TV Viewing Times } & \multicolumn{2}{|c|}{ Computer Usage Times } & \multicolumn{2}{|c|}{ Mobile Phone Viewings } \\
\hline & $\leq 2 \mathrm{~h} / \mathrm{day}$ & $>2$ h/day & $\leq 2 \mathrm{~h} /$ day & $>2$ h/day & $\leq 1 \mathrm{~h} /$ day & $>1$ h/day \\
\hline \multicolumn{7}{|l|}{ Age } \\
\hline 18-25 years old & 481 (77.1) & $143(22.9)$ & $528(84.6)$ & $96(15.4)$ & $217(34.8)$ & $407(65.2)$ \\
\hline 26-35 years old & $1187(74.4)$ & $408(25.6)$ & $1228(77.0)$ & $367(23.0)$ & $614(38.5)$ & $981(61.5)$ \\
\hline $36-45$ years old & $89(70.6)$ & $37(29.4)$ & $107(84.9)$ & $19(15.1)$ & $47(37.3)$ & $79(62.7)$ \\
\hline \multicolumn{7}{|l|}{ Parity } \\
\hline Pregnant women in first pregnancy & $1299(74.0)$ & $456(26.0)$ & $1350(76.9)$ & $405(23.1)$ & $674(38.4)$ & $1081(61.6)$ \\
\hline $\begin{array}{l}\text { Pregnant women in second } \\
\text { pregnancy }\end{array}$ & $458(77.6)$ & $132(22.4)$ & $513(86.9)$ & $77(13.1)$ & $204(34.6)$ & $386(65.4)$ \\
\hline \multicolumn{7}{|l|}{ Hospital level } \\
\hline Level 3A hospital & $1353(74.2)$ & $471(25.8)$ & $1418(77.7)$ & $406(22.3)$ & $700(38.4)$ & $1124(61.6)$ \\
\hline Level 2 A hospitals & $248(79.7)$ & $63(20.3)$ & $280(90.0)$ & $31(10.0)$ & $103(33.1)$ & $208(66.9)$ \\
\hline Level 2B hospitals and below & $156(74.3)$ & $54(25.7)$ & $165(78.6)$ & $45(21.4)$ & $75(35.7)$ & $135(64.3)$ \\
\hline \multicolumn{7}{|l|}{ Nationality } \\
\hline Han nationality & $1684(74.8)$ & $568(25.2)$ & $1783(79.2)$ & $469(20.8)$ & $845(37.5)$ & $1407(62.5)$ \\
\hline Non-Han nationality & $73(78.5)$ & $20(21.5)$ & $80(86.0)$ & $13(14.0)$ & $33(35.5)$ & $60(64.5)$ \\
\hline \multicolumn{7}{|l|}{ Without siblings } \\
\hline Yes & $806(77.1)$ & $240(22.9)$ & $1029(79.2)$ & $270(20.8)$ & 487 (37.5) & $812(62.5)$ \\
\hline No & $951(73.2)$ & $348(26.8)$ & $834(79.7)$ & $212(20.3)$ & $391(37.4)$ & $655(62.6)$ \\
\hline \multicolumn{7}{|l|}{ Husband without siblings } \\
\hline Yes & $887(75.6)$ & $286(24.4)$ & $942(80.4)$ & $230(19.6)$ & $422(36.0)$ & $750(64.0)$ \\
\hline No & $870(74.2)$ & $302(25.8)$ & $921(78.5)$ & $252(21.5)$ & $456(38.9)$ & $717(61.1)$ \\
\hline \multicolumn{7}{|l|}{ Marital status } \\
\hline Unmarried & $30(61.2)$ & 19 (38.8) & 39 (79.6) & $10(20.4)$ & $25(51.0)$ & $24(49.0)$ \\
\hline Primary marriage & $1659(75.2)$ & $546(24.8)$ & $1746(79.2)$ & $459(20.8)$ & $820(37.2)$ & $1385(62.8)$ \\
\hline Remarried & $51(72.9)$ & $19(27.1)$ & $59(84.3)$ & $11(15.7)$ & $23(32.9)$ & $47(67.1)$ \\
\hline Divorced or Widowed & $17(81.0)$ & $4(19.0)$ & $19(90.5)$ & $2(9.5)$ & $10(47.6)$ & $11(52.4)$ \\
\hline
\end{tabular}


Table 3. Cont.

\begin{tabular}{|c|c|c|c|c|c|c|}
\hline \multirow{2}{*}{ Variable } & \multicolumn{2}{|c|}{ TV Viewing Times } & \multicolumn{2}{|c|}{ Computer Usage Times } & \multicolumn{2}{|c|}{ Mobile Phone Viewings } \\
\hline & $\leq 2 \mathrm{~h} /$ day & $>2 \mathrm{~h} /$ day & $\leq 2 \mathrm{~h} / \mathrm{day}$ & $>2$ h/day & $\leq 1 \mathrm{~h} /$ day & $>1$ h/day \\
\hline Education level & & & & & & \\
\hline Basic education & $290(72.1)$ & $112(27.9)$ & $371(92.3)$ & $31(7.7)$ & $134(33.3)$ & $268(66.7)$ \\
\hline Secondary education & $262(74.0)$ & $92(26.0)$ & $316(89.3)$ & $38(10.7)$ & $130(36.7)$ & $224(63.3)$ \\
\hline Higher education & $1205(75.8)$ & $384(24.2)$ & $1176(74.0)$ & $413(26.0)$ & $614(38.6)$ & $975(61.4)$ \\
\hline \multicolumn{7}{|l|}{ Residence } \\
\hline Urban & $1414(75.2)$ & $466(24.8)$ & $410(88.2)$ & $55(11.8)$ & $170(36.6)$ & 295 \\
\hline Rural & $343(73.8)$ & $122(26.2)$ & $1453(77.3)$ & $427(22.7)$ & $708(37.7)$ & $1172(62.3)$ \\
\hline \multicolumn{7}{|l|}{$\begin{array}{l}\text { The per capital monthly income of } \\
\text { the family }\end{array}$} \\
\hline$<¥ 4500$ & 467 (76.4) & $144(23.6)$ & $503(82.3)$ & $108(17.7)$ & $234(38.3)$ & $377(61.7)$ \\
\hline$¥ 4500$ and $¥ 9000$ & $759(76.7)$ & $230(23.3)$ & $809(81.8)$ & $180(18.2)$ & $373(37.7)$ & $616(62.3)$ \\
\hline$>¥ 9000$ & $531(71.3)$ & $214(28.7)$ & $551(74.0)$ & $194(26.0)$ & $271(36.4)$ & $474(63.6)$ \\
\hline \multicolumn{7}{|l|}{ Occupation } \\
\hline Rural migrant workers & $91(77.1)$ & $27(22.88)$ & 115 (97.5) & $3(2.5)$ & $53(44.9)$ & $65(55.1)$ \\
\hline $\begin{array}{l}\text { Urban and rural unemployed } \\
\text { Industrial workers of }\end{array}$ & $388(70.2)$ & $165(29.8)$ & $491(88.8)$ & $62(11.2)$ & $204(36.9)$ & $349(63.1)$ \\
\hline $\begin{array}{l}\text { Non-agricultural registered } \\
\text { permanent residence }\end{array}$ & $39(78.0)$ & $11(22.0)$ & $35(70.0)$ & $15(30.0)$ & $20(40.0)$ & $30(60.0)$ \\
\hline Individual business & $146(73.4)$ & $53(26.6)$ & $179(89.9)$ & $20(10.1)$ & $64(32.2)$ & $135(67.8)$ \\
\hline Business services staff & $119(76.8)$ & $36(23.2)$ & $125(80.7)$ & $30(19.3)$ & $60(38.7)$ & $95(61.3)$ \\
\hline Civil servants & $324(81.4)$ & $74(18.6)$ & $293(73.6)$ & $105(26.4)$ & $153(38.4)$ & $245(61.6)$ \\
\hline $\begin{array}{l}\text { Senior managers and Middle-level } \\
\text { managers in large and medium } \\
\text { enterprises }\end{array}$ & $78(81.3)$ & $18(18.7)$ & $64(66.7)$ & $32(33.3)$ & $39(40.6)$ & $57(59.4)$ \\
\hline Private entrepreneur & $59(67.8)$ & $28(32.2)$ & $73(83.9)$ & $14(16.1)$ & $21(24.1)$ & $66(75.9)$ \\
\hline Professionals & $186(76.2)$ & $58(23.8)$ & $175(71.7)$ & $69(28.3)$ & $86(35.3)$ & $158(64.7)$ \\
\hline Clerk & $106(76.3)$ & $33(23.7)$ & $78(56.1)$ & $61(43.9)$ & $53(38.1)$ & $86(61.9)$ \\
\hline Students & $15(100.0)$ & $0(0.0)$ & $9(60.0)$ & $6(40.0)$ & $4(26.7)$ & $11(73.3)$ \\
\hline Other & $206(70.8)$ & $85(29.2)$ & $226(77.7)$ & $65(22.3)$ & $121(41.6)$ & $170(58.4)$ \\
\hline \multicolumn{7}{|l|}{ Occupational category } \\
\hline Non-manual & $914(77.6)$ & $264(22.4)$ & $871(73.9)$ & $307(26.1)$ & $420(35.7)$ & $758(64.4)$ \\
\hline Manual & $249(77.1)$ & $74(22.9)$ & $275(85.1)$ & $48(14.9)$ & $133(41.2)$ & $190(58.8)$ \\
\hline Unemployed & $388(70.2)$ & $165(29.8)$ & $491(88.8)$ & $62(11.2)$ & $204(36.9)$ & $349(63.1)$ \\
\hline Others & $206(70.8)$ & $85(29.2)$ & $226(77.7)$ & $65(22.3)$ & $121(41.6)$ & $170(58.4)$ \\
\hline \multicolumn{7}{|l|}{ Trimester of pregnancy } \\
\hline First trimester & $216(73.7)$ & $77(26.3)$ & $232(79.2)$ & $61(20.8)$ & $119(40.6)$ & $174(59.4)$ \\
\hline Second trimester & $540(77.0)$ & $161(23.0)$ & $564(80.5)$ & $137(19.5)$ & $274(39.1)$ & $427(60.9)$ \\
\hline Third trimester & $1001(74.1)$ & $350(25.9)$ & $1067(79.0)$ & $284(21.0)$ & $485(35.9)$ & $866(64.1)$ \\
\hline
\end{tabular}

Prolonged computer usage times were reported by 405 (23.1\%) and 77 (13.1\%) participants on their first and second pregnancies. A total of $55(11.8 \%)$ and $427(22.7 \%)$ participants from urban and rural areas, respectively, were reported to watch computer more than $2 \mathrm{~h}$ daily (see Table 3 ).

Prolonged mobile phone usage times were reported by $1081(61.6 \%)$ and $386(65.4 \%)$ participants on their first and second pregnancies. A total of 1172 (62.3\%) and 295 (63.4\%) participants from urban and rural areas, respectively, were reported to watch over one hour of mobile phone per day (see Table 3).

\subsection{Generalized Linear Model for Long TV, Computer, Mobile and Total Viewing Time among Pregnant Women, China}

Generalized linear model found that pregnant women in their second pregnancy $(p=0.0009)$ had high level of education $(p=0.008)$ and were less likely have long TV viewing times. Pregnant women whose family's per capital monthly income was more than $¥ 9000(p=0.020)$ and those who were $26-35$ years old $(p=0.007)$ and $36-45$ years old $(p=0.026)$ tended to have long TV viewing times.

Generalized linear model indicated that pregnant women in their second pregnancy $(p<0.001)$ from level 2A hospitals $(p=0.005)$, of manual $(p=0.040)$ and unemployed $(p<0.001)$, were less likely 
to have long computer usage times. Pregnant women with high level of education $(p=0.0002)$ and those aged $26-35$ years old $(p=0.010)$ tended to have long computer usage times.

Generalized linear model found that pregnant women of manual $(p=0.001)$, unemployed $(p=0.012)$, and with high level of education $(p=0.047)$ were less likely to have long mobile phone viewing times. Pregnant women in their second pregnancy $(p=0.041)$ tended to have long mobile phone viewing times.

Generalized linear model showed that pregnant women in their second pregnancy $(p=0.006)$, manual $(p=0.002)$, and unemployed $(p=0.0001)$ were less likely have long screen times. Pregnant women of primary marriage $(p=0.008)$ and remarried $(p=0.018)$ tended to have long screen viewing times (see Table 4). 
Table 4. Generalized linear model for identifying factors that affect TV viewing times, computer viewing times, mobile viewing times, and total viewing time among pregnant women, China, 2015.

\begin{tabular}{|c|c|c|c|c|c|c|c|c|}
\hline \multirow{2}{*}{ Parameter } & \multicolumn{2}{|c|}{ TV Viewing Times } & \multicolumn{2}{|c|}{ Computer Usage Times } & \multicolumn{2}{|c|}{ Mobile Phone Usage Times } & \multicolumn{2}{|c|}{ The Total Time } \\
\hline & Estimate $(S E)$ & $p$ & Estimate (SE) & $p$ & Estimate (SE) & $p$ & Estimate $(S E)$ & $p$ \\
\hline TV viewing times & - & - & $-0.059(0.028)$ & 0.035 & $0.134(0.027)$ & $<0.001$ & - & - \\
\hline Computer viewing times & $-0.032(0.015)$ & 0.035 & - & - & $0.049(0.020)$ & 0.015 & - & - \\
\hline Mobile phone usage times & $0.080(0.016)$ & $<0.001$ & $0.053(0.022)$ & 0.015 & -2 & - & - & - \\
\hline Pregnant women in second pregnancy vs. Pregnant women in first pregnancy & $-0.288(0.087)$ & 0.0009 & $-0.461(0.117)$ & $<0.001$ & $0.230(0.113)$ & 0.041 & $-0.523(0.190)$ & 0.006 \\
\hline Level 3A hospital vs. Level 2B hospitals and below & $0.118(0.123)$ & 0.339 & $-0.027(0.166)$ & 0.869 & $-0.066(0.160)$ & 0.681 & $0.026(0.272)$ & 0.924 \\
\hline Level 2A hospitals vs. Level 2B hospitals and below & $-0.187(0.152)$ & 0.220 & $-0.577(0.205)$ & 0.005 & $0.155(0.197)$ & 0.431 & $-0.616(0.335)$ & 0.066 \\
\hline Manual vs. Non-manual & $0.103(0.113)$ & 0.360 & $-0.312(0.152)$ & 0.040 & $-0.502(0.146)$ & 0.001 & $-0.778(0.248)$ & 0.002 \\
\hline Unemployed vs. Non-manual & $0.270(0.095)$ & 0.005 & $-0.722(0.128)$ & $<0.001$ & $-0.312(0.124)$ & 0.012 & $-0.798(0.208)$ & 0.0001 \\
\hline Others vs. Non-manual & $0.229(0.110)$ & 0.038 & $-0.257(0.149)$ & 0.084 & $-0.230(0.143)$ & 0.109 & $-0.274(0.243)$ & 0.259 \\
\hline Non-Han nationality vs. Han nationality & $-0.161(0.178)$ & 0.366 & $-0.175(0.240)$ & 0.467 & $0.191(0.231)$ & 0.409 & $-0.137(0.393)$ & 0.728 \\
\hline Without siblings vs. With siblings & $-0.102(0.072)$ & 0.157 & $-0.105(0.097)$ & 0.282 & $0.059(0.094)$ & 0.529 & $-0.151(0.159)$ & 0.342 \\
\hline Husband was not single child vs. Husband was single child & $-0.041(0.071)$ & 0.565 & $-0.076(0.096)$ & 0.431 & $0.013(0.092)$ & 0.889 & $-0.107(0.157)$ & 0.495 \\
\hline Primary marriage vs. Divorced or Widowed & $0.585(0.368)$ & 0.112 & $0.560(0.496)$ & 0.259 & $0.831(0.477)$ & 0.082 & $2.160(0.810)$ & 0.008 \\
\hline Unmarried vs. Divorced or Widowed & $0.692(0.436)$ & 0.113 & $0.791(0.588)$ & 0.179 & $0.138(0.566)$ & 0.808 & $1.720(0.961)$ & 0.074 \\
\hline Remarried vs. Divorced or Widowed & $0.446(0.416)$ & 0.285 & $0.473(0.564)$ & 0.402 & $1.060(0.542)$ & 0.051 & $2.180(0.920)$ & 0.018 \\
\hline Secondary education vs. Basic education & $-0.117(0.124)$ & 0.343 & $0.094(0.167)$ & 0.573 & $-0.024(0.161)$ & 0.880 & $-0.060(0.273)$ & 0.827 \\
\hline Higher education vs. Basic education & $-0.296(0.111)$ & 0.008 & $0.553(0.150)$ & 0.0002 & $-0.287(0.144)$ & 0.047 & $-0.087(0.244)$ & 0.723 \\
\hline Urban vs. Rural & $-0.077(0.095)$ & 0.421 & $0.082(0.129)$ & 0.523 & $0.002(0.124)$ & 0.986 & $0.002(0.210)$ & 0.991 \\
\hline$¥ 4500$ and $¥ 9000$ vs. $<¥ 4500$ & $0.046(0.090)$ & 0.604 & $-0.215(0.121)$ & 0.075 & $-0.100(0.116)$ & 0.388 & $-0.283(0.197)$ & 0.151 \\
\hline$>¥ 9000$ vs. $<¥ 4500$ & $0.232(0.099)$ & 0.020 & $0.014(0.135)$ & 0.919 & $-0.089(0.129)$ & 0.490 & $0.166(0.220)$ & 0.450 \\
\hline Second trimester vs. First trimester & $0.033(0.116)$ & 0.779 & $0.024(0.157)$ & 0.877 & $-0.083(0.151)$ & 0.585 & $-0.034(0.257)$ & 0.896 \\
\hline Third trimester vs. First trimester & $0.097(0.108)$ & 0.368 & $-0.004(0.146)$ & 0.977 & $0.088(0.140)$ & 0.528 & $0.203(0.238)$ & 0.394 \\
\hline $26-35$ years old vs. $18-25$ years old & $0.225(0.084)$ & 0.007 & $0.293(0.114)$ & 0.010 & $-0.217(0.109)$ & 0.047 & $0.298(0.185)$ & 0.109 \\
\hline $36-45$ years old vs. $18-25$ years old & $0.395(0.177)$ & 0.026 & $0.270(0.239)$ & 0.259 & $-0.275(0.230)$ & 0.233 & $0.394(0.391)$ & 0.314 \\
\hline
\end{tabular}




\subsection{Multivariate Logistic Regression Model for TV Viewing Times of More Than Two Hours Daily}

Pregnant women with more than one hour of mobile phone usage time were more likely to watch over two hours of TV per day than pregnant women with less than one hour of mobile phone usage $[O R=1.52,95 \% C I: 1.25-1.86]$. Unemployed pregnant women [OR $=1.65,95 \% C I: 1.30-2.10]$ and marked others [OR $=1.42,95 \%$ CI: $1.064-1.89]$ tended to watch over two hours of TV daily. Pregnant women without siblings were less likely to have prolonged TV viewing times than those with siblings $[O R=0.79,95 \%$ CI: 0.65-0.95]. Pregnant women whose family's per capita income was more than $¥ 9000$ were likely watch over two hours of TV per day [OR $=1.42,95 \%$ CI: $1.10-1.86]$ (see Table 5).

\subsection{Multivariate Logistic Model for Computer Usage Times of More Than Two Hours per Day}

Pregnant women in their second pregnancy were less likely to have over two hours of computer viewing time per day than those in their first pregnancy [OR $=0.56,95 \%$ CI: $0.42-0.74]$. Pregnant women from Level 2A hospitals tended to have over two hours of computer viewing time per day $[O R=0.53,95 \%$ CI: 0.31-0.89]. Unemployed pregnant women [OR $=0.51,95 \%$ CI: $0.37-0.70]$ were less likely to have over two hours of computer viewing time daily. Pregnant women with completed high education tended to have computer viewing times of over two hours per day $[O R=2.72,95 \% C I$ : 1.78-4.16]. Pregnant women without siblings were less likely to have prolonged computer viewing times than those with siblings [OR $=0.76,95 \%$ CI: 0.61-0.94]. Pregnant women whose family's per capita income was between $¥ 4500$ and $¥ 9000$ were less likely have over two hours of computer viewing time per day $[O R=0.69,95 \%$ CI: $0.52-0.92]$ (see Table 5).

\subsection{Multivariate Logistic Model for Mobile Phone Usage Times of More Than One Hour Daily}

Pregnant women with more than two hours of TV viewing time were more likely to have over one hour of mobile phone viewing time per day than those with less than two hours of TV viewing time $[O R=1.56,95 \% C I: 1.27-1.91]$. Pregnant women aged between 26 to 35 years tended to have over one hour of mobile phone viewing time per day [OR $=0.80,95 \% C I$ : $0.65-0.98]$. Pregnant women in their second pregnancy were more likely to have prolonged mobile phone viewing times than those in their first pregnancy $(O R=1.25,95 \%$ CI: $1.01-1.55)$ (see Table 5). 
Table 5. Multivariate logistic regression model for prolonged TV, computer, and mobile viewing times among pregnant women, China, 2015.

\begin{tabular}{|c|c|c|c|c|c|}
\hline \multicolumn{2}{|l|}{ TV Viewing Times } & \multicolumn{2}{|l|}{ Computer Usage Times } & \multicolumn{2}{|l|}{ Mobile Phone Usage Times } \\
\hline Parameter & $O R^{\mathrm{a} 1}(95 \% C I)$ & Parameter & $O R^{\mathrm{a} 2}(95 \% C I)$ & Parameter & $O R^{\mathrm{a} 3}(95 \% C I)$ \\
\hline Mobile phone usage time & & Mobile phone usage time & & TV viewing time & \\
\hline$\leq 1 \mathrm{~h}$ & 1.00 & $\leq 1 \mathrm{~h}$ & 1.00 & $\leq 2 \mathrm{~h}$ & 1.00 \\
\hline$>1 \mathrm{~h}$ & $1.52(1.25-1.86)$ & $>1 \mathrm{~h}$ & $1.32(1.06-1.64)$ & $>2 \mathrm{~h}$ & $1.56(1.27-1.91)$ \\
\hline Hospital level & & Hospital level & & Computer viewing time & \\
\hline Level 2B hospitals and below & 1.00 & Level 2B hospitals and below & 1.00 & $\leq 2 \mathrm{~h}$ & 1.00 \\
\hline Level 3A hospital & $1.11(0.80-1.56)$ & Level 3A hospital & $0.88(0.61-1.27)$ & $>2 \mathrm{~h}$ & $1.32(1.06-1.65)$ \\
\hline Level 2 A hospitals & $0.76(0.50-1.17)$ & Level 2 A hospitals & $0.53(0.31-0.89)$ & - & - \\
\hline Occupational category & & Occupational category & & Occupational category & \\
\hline Non-manual & 1.00 & Non-manual & 1.00 & Non-manual & 1.00 \\
\hline Manual & $1.17(0.86-1.58)$ & Manual & $0.74(0.52-1.06)$ & Manual & $0.72(0.55-0.94)$ \\
\hline Unemployed & $1.65(1.30-2.10)$ & Unemployed & $0.51(0.37-0.70)$ & Unemployed & $0.84(0.67-1.05)$ \\
\hline Others & $1.42(1.06-1.89)$ & Others & $0.86(0.62-1.18)$ & Others & $0.74(0.56-0.96)$ \\
\hline Without siblings & & Without siblings & & Without siblings & - \\
\hline No & 1.00 & No & 1.00 & No & - \\
\hline Yes & $0.79(0.65-0.95)$ & Yes & $0.76(0.61-0.94)$ & Yes & - \\
\hline The per capital monthly income of the family & & The per capital monthly income of the family & & The per capital monthly income of the family & - \\
\hline$<4500 ¥$ & 1.00 & $<4500 ¥$ & 1.00 & $<4500 ¥$ & - \\
\hline $4500 ¥$ and $9000 ¥$ & $1.03(0.81-1.32)$ & $4500 ¥$ and $9000 ¥$ & $0.69(0.52-0.92)$ & $4500 ¥$ and $9000 ¥$ & - \\
\hline$>9000 ¥$ & $1.42(1.09-1.86)$ & $>9000 ¥$ & $0.92(0.68-1.23)$ & $>9000 ¥$ & - \\
\hline Parity & - & Parity & & Parity & \\
\hline Pregnant women in first pregnancy & - & Pregnant women in first pregnancy & 1.00 & Pregnant women in first pregnancy & 1.00 \\
\hline Pregnant women in second pregnancy & - & Pregnant women in second pregnancy & $0.56(0.42-0.74)$ & Pregnant women in second pregnancy & $1.25(1.01-1.55)$ \\
\hline Education level & - & Education level & & Education level & \\
\hline Basic education & - & Basic education & 1.00 & Basic education & 1.00 \\
\hline Secondary education & - & Secondary education & $1.34(0.81-2.23)$ & Secondary education & $0.83(0.61-1.13)$ \\
\hline Higher education & - & Higher education & $2.72(1.78-4.16)$ & Higher education & $0.73(0.56-0.95)$ \\
\hline Age & - & Age & & Age & \\
\hline $18-25$ years old & - & $18-25$ years old & 1.00 & $18-25$ years old & 1.00 \\
\hline $26-35$ years old & - & $26-35$ years old & $1.33(1.02-1.74)$ & $26-35$ years old & $0.80(0.65-0.98)$ \\
\hline $36-45$ years old & - & $36-45$ years old & $1.09(0.60-1.95)$ & $36-45$ years old & $0.70(0.45-1.08)$ \\
\hline Marital status & - & Marital status & - & Marital status & \\
\hline Primary marriage & - & Primary marriage & - & Primary marriage & 1.00 \\
\hline Unmarried & - & Unmarried & - & Unmarried & $0.48(0.27-0.85)$ \\
\hline Remarried & - & Remarried & - & Remarried & $1.14(0.67-1.92)$ \\
\hline Divorced or Widowed & - & Divorced or Widowed & - & Divorced or Widowed & $0.66(0.28-1.58)$ \\
\hline Residence & - & Residence & - & Residence & - \\
\hline Rural & - & Rural & 1.00 & Rural & - \\
\hline Urban & - & Urban & $1.34(0.97-1.87)$ & Urban & - \\
\hline
\end{tabular}

${ }^{a 1}$ Adjusted for mobile phone usage times, hospital level, occupational category, without siblings, and the per capita monthly income of the family; ${ }^{\text {a2 }}$ Adjusted for mobile phone usage times, age, hospital level, occupational category, without siblings, education level, residence, the per capita monthly income of the family, and trimester of pregnancy; ${ }^{3}$ Adjusted for TV viewing times, computer usage times, age, occupational category, marital status, education level, and trimester of pregnancy. 


\section{Discussion}

Prolonged television, computer, and mobile phone viewing times are common during pregnancy. Clarifying the factors affecting screen-viewing times during pregnancy is necessary to decrease it through educational behavior change interventions. A previous national study found that among Chinese women, prolonged television viewing time prevalence was high (69.3\%) in 2002 [13] and 2013-2015 (62.63\%) [12], and these prevalence rates are higher than those among pregnant women of the present study; however, the prevalence rate of the present study still has a high number for pregnant women and may increase the number of adverse pregnancy outcomes. On 29 October 2015, the Chinese government announced the "two-child policy", implying that more Chinese women will be impregnated. The present study found that high-risk groups for prolonged mobile phone viewing were those in their second pregnancy, although they were less likely have prolonged computer viewing times. Further measures are necessary to achieve the target of Health China 2030, which promotes healthy lifestyles and eliminates health-related risk factors [33]. This study has implications in the implementation and enforcement of Health China 2030 that promotes healthy lifestyles and eliminates health-related risk factors. An American family-based intervention in women, infants, and children [34] may indicate that a family-based intervention of increasing outdoor playtime may be also feasible and efficient among pregnant women.

The present findings indicated a mutual correlation between prolonged TV and mobile phone viewing times. Pregnant women with prolonged mobile phone viewing times were more likely to have prolonged TV viewing times. A similar study also found that pregnant women who used both mobile phones and computers were more likely to have a short pregnancy duration [2]. This result may indicate that pregnant women with prolonged mobile usage have a more positive attitude towards electronic products including television compared with those without prolonged mobile phone usage times. This finding may be associated with the multiple and advanced functions of TV nowadays, whereby people can watch films, TV series, live online shows, and even play games [35]. Pregnant women can enjoy similar experiences and advanced features through mobile phone and TV, such as watching movies and popular soap operas to spend daily leisure time. As applications that can connect to and control TV are continuously being developed, pregnant women can use mobile phones when watching TV, which may also increase the likelihood of prolonged TV viewing times of pregnant women who enjoy using mobile phones. Thus, the effect of the screen viewing of various electronic products should be investigated in the future. Pregnant women with prolonged TV or mobile phone viewing times tended to suffer greater adverse health outcomes. Moreover, pregnant women whose single screen viewing times on one of the modalities were not prolonged may have a prolonged combined total viewing time.

A high-risk group with prolonged mobile phone viewing times is those women in their second pregnancy. Interestingly, they are also less likely to have prolonged computer viewing times. Decreased phone usage among pregnant women during their first pregnancy may be attributed to them being cautious and to their families who were likely to reduce mobile phone usage. Thus, the great potential need for surfing the Internet to seek pregnancy-related knowledge and other online information may increase possibility of computer viewing [36]. However, pregnant women in their second pregnancy with prior gestational experience may not be as cautious as those in their first pregnancy, including the reduced frequency of prenatal examination [37]. A previous study found a high rate of information seeking behaviors through the Internet given that $88.7 \%$ of pregnant women used the Internet to learn about health-related information, in which fetal development and pregnancy nutrition knowledge were the two most often mentioned topics [36]. Additionally, pregnant women in their first pregnancy were usually younger than those in their second pregnancy, and given that younger women viewed computers for over two hours more often in China [38], this may explain the finding from another perspective.

Pregnant women with siblings tended to have prolonged TV and computer viewing times. This scenario may indicate the positive influence of siblings on pregnant women in prolonged TV 
and computer viewing times, and a previous study has also found the influence of siblings on the insufficient sleep of pregnant women in China [39]. Future studies should focus on pregnant women with siblings and explore the influence of siblings on their family members regarding prolonged screen viewing times and other related health behaviors. Prolonged screen viewing times are definitely unhealthy, but we cannot deny their convenience and instantaneity, and a technology-based e-health intervention may be convenient and efficient at reducing the frequency of prolonged TV and computer viewing times [40], for example, daily health message and timing reminder software.

Furthermore, women with high monthly income and who were unemployed showed an increased prevalence of prolonged TV viewing times during pregnancy. Pregnant women with high income likely have prolonged television viewing times. Similar to a previous Singaporean study, pregnant women with high incomes are likely have high sitting times, including watching TV and low reduction in physical activity [41]. Those with high monthly income possibly have low economic pressure and less housework. Likewise, pregnant women with high monthly income have more money to hire nannies and are more likely to stay at home for safety, giving them sufficient time to watch TV. By contrast, pregnant women with low monthly income cater to their family or work for more money, which may explain this finding. The present study also found that unemployed pregnant women likely watched television for long periods of time. The possible reason is that those pregnant women need more time to use television for leisure time. With the rise of Chinese economic levels, the number of pregnant women with high monthly income will further increase. Health professionals should focus on these pregnant women, and measures should also be taken to intervene in the prolonged TV watching times.

Additionally, pregnant women with high educational level aged 26-35 years old show increased prevalence of prolonged computer viewing times. Pregnant women with complete higher education likely watch computers for over two hours per day, and a previous national survey in China found higher prevalence rate of computer usage among women from urban areas than those from rural areas [42]. Most pregnant women who are senior intellectuals may be mental workers, and possibly have higher acceptance and usage habits in general life and work for computer usage compared with pregnant women with low educational level. Additionally, those pregnant women may undertake significant business in their institutions and whether or not they needed the assistance of computers demands further attention. A previous Chinese study also found that pregnant women with high education level regarded health-related information from the Internet as believable and dependable [36]. This previous finding may indicate that pregnant women with higher educational level have high frequencies of information-seeking behavior through computers. The present finding may reveal a key situation that pregnant women of higher educational level tended to have high possibility of prolonged computer viewing times. Health-related workers and future studies must focus on those pregnant women, and other populations with higher educational level may also need investigation. Family-work unit-based interventions may be necessary for those pregnant women. Pregnant women with siblings were more likely to have prolonged TV viewing times and prolonged computer viewing times than those without siblings, which may indicate the influence of siblings on prolonged TV and computer viewing times. Reasons for the present finding are multifaceted and synthetic; thus, future studies should focus on this phenomenon. In any case, given the issue of "two-child policy", families adhering to this policy will increasingly appear in China, and the present finding will be worse than before. Efficient preventive measures should be taken to reduce the prevalence among pregnant women that have siblings.

Pregnant women with basic educational level, low age, and primary marriage showed an increased prevalence of prolonged mobile phone viewing times. Pregnant women with basic educational level and who are younger presented more chances of prolonged mobile phone viewing times. Furthermore, previous research indicates that young women with low education tended to spend long time on each call [19], and a Japanese survey also showed that young pregnant women with low educational level likely use their mobile phones excessively [1]. Young pregnant women are 
possibly intrigued by mobile phones. Besides, pregnant women with basic educational level may have less amateur activities for their limited knowledge and opportunities compared with those of general educational level. Pregnant women with excessive mobile phone usage prefer to hide their phones in their pockets or other places for convenience [1]; thus, those pregnant women may be exposed to increased radiation. The present study also found that unmarried pregnant women were less likely to have prolonged mobile phone usage, which differs from a previous study in which single pregnant women tended to have excessive usage of mobile phones [1]. Thus, further studies should be based on ethnic, regional, and socioeconomic status to confirm the high risk population in China.

The present study also has certain notable limitations. First, the cross-sectional survey data prevented researchers from making direct causal inferences, exploring whether or not the unmeasured factors may explain the observed relationships, and determining the direction of causality. Second, the face-to-face survey administration design and self-reported screen-viewing times may have introduced some information and measurement bias. Third, our study is not precisely nationally representative. The sample comprised the population of pregnant women in five regions of China, namely, Chongqing, Chengdu, Zunyi, Liaocheng, and Tianjin. Chongqing, Chengdu, and Zunyi are in southern China, while Liaocheng and Tianjin are in northern China. No evident difference is observed between western, eastern, and central China relative to the proportion of pregnant women that watch TV [43].

\section{Conclusions}

Pregnant women have high a prevalence rate of prolonged TV, computer, and mobile phone viewing times, which is common for those in their first and second pregnancies. Findings provide a primary and exploratory understanding of the prolonged television, computer, and mobile phone viewing times among Chinese pregnant women. This study outlines some of the implications of decreasing screen-viewing times during pregnancy in China, and these findings may concentrate on the implementation and development of the "Healthy China 2030 policy".

Acknowledgments: This project was supported by the Medjaden Academy \& Research Foundation for Young Scientists (Grant No. MJR20150047) and Summer Social Practice Project of School of Public Health and Management, Chongqing Medical University. We thank team members for their effort and contributions to this study.

Author Contributions: All authors contributed to the design of the study. Xianglong Xu analyzed the data, data interpretation, and drafted the manuscript. Dengyuan Liu collected data, contributed to data analysis, data interpretation, and drafted the manuscript. Yunshuang Rao collected data and helped draft the manuscript. Huan Zeng, Fan Zhang, Lu Wang, Yaojie Xie, Manoj Sharma, and Yong Zhao helped draft the manuscript. All authors have read and approved of the final manuscript. All authors have seen and approved of the final version of the manuscript.

Conflicts of Interest: The authors declare no conflict of interest.

\section{References}

1. Lu, X.; Oda, M.; Ohba, T.; Mitsubuchi, H.; Masuda, S.; Katoh, T. Association of excessive mobile phone use during pregnancy with birth weight: an adjunct study in Kumamoto of Japan Environment and Children's Study. Environ. Health Prev. Med. 2017, 22, 52. [CrossRef] [PubMed]

2. Col-Araz, N. Evaluation of factors affecting birth weight and preterm birth in southern turkey. J. Pak. Med. Assoc. 2013, 63, 459-462. [PubMed]

3. Dempsey, P.C.; Howard, B.J.; Lynch, B.M.; Owen, N.; Dunstan, D.W. Associations of television viewing time with adults' well-being and vitality. Prev. Med. 2014, 69, 69-74. [CrossRef] [PubMed]

4. Thorp, A.A.; Mcnaughton, S.A.; Owen, N.; Dunstan, D.W. Independent and joint associations of TV viewing time and snack food consumption with the metabolic syndrome and its components; A cross-sectional study in Australian adults. Int. J. Behav. Nutr. Phys. Act. 2013, 10, 11. [CrossRef] [PubMed]

5. Xie, Y.J.; Stewart, S.M.; Lam, T.H.; Viswanath, K.; Chan, S.S. Television viewing time in Hong Kong adult population: Associations with body mass index and obesity. PLoS ONE 2014, 9, e85440. [CrossRef] [PubMed] 
6. Rockette-Wagner, B.; Edelstein, S.; Venditti, E.M.; Reddy, D.; Bray, G.A.; Carrionpetersen, M.L.; Dabelea, D.; Delahanty, L.M.; Florez, H.; Franks, P.W. The impact of lifestyle intervention on sedentary time in individuals at high risk of diabetes. Diabetologia 2015, 58, 1198-1202. [CrossRef] [PubMed]

7. Salanave, B.; Vernay, M.; Deschamps, V.; Malon, A.; Oléko, A.; Hercberg, S.; Castetbon, K. Television viewing duration and blood pressure among 18-74-year-old adults. The French nutrition and health survey (ENNS, 2006-2007). J. Sci. Med. Sport 2016, 19, 738-743. [CrossRef] [PubMed]

8. Ivy, S. Modeling indoor TV/screen viewing and adult physical and mental health: Health survey for England, 2012. Environ. Sci. Pollut. Res. 2016, 23, 11708-11715.

9. Costigan, S.A.; Barnett, L.; Plotnikoff, R.C.; Lubans, D.R. The health indicators associated with screen-based sedentary behavior among adolescent girls: A systematic review. J. Adolesc. Health 2013, 52, 382-392. [CrossRef] [PubMed]

10. Shen, Y. Mobile Phone Use Behavior and Its Influencing Factors. Ph.D. Thesis, Zhejiang University, Hangzhou, China, 2009. (In Chinese)

11. Lönn, S.; Ahlbom, A.; Christensen, H.C.; Johansen, C.; Schüz, J.; Edström, S.; Henriksson, G.; Lundgren, J.; Wennerberg, J.; Feychting, M. Mobile phone use and risk of parotid gland tumor. Am. J. Epidemiol. 2006, 164, 637-643. [CrossRef] [PubMed]

12. Xue, H.M.; Liu, Q.Q.; Tian, G.; Quan, L.M.; Zhao, Y.; Cheng, G. Television watching and telomere length among adults in southwest China. Am. J. Public Health 2017, 109, 1425-1432. [CrossRef] [PubMed]

13. Ma, G.S.; Liu, A.L.; Cui, Z.H.; Li, Y.P.; Hu, X.Q.; Luan, D.C. The situation of television viewing of people in China. Chin. J. Health Educ. 2006, 22, 167-170. (In Chinese)

14. Qian, Y.; Wang, B.B.; Huang, N.; Zhao, X.G.; Duan, X.L.; Jiang, Y.; Wang, L.M.; Guo, J. Investigation of computer and mobile phone-related radiation exposure time of Chinese adults. J. Environ. Health 2014, 31, 974-977. (In Chinese)

15. Dennison, B.A.; Erb, T.A.; Jenkins, P.L. Television viewing and television in bedroom associated with overweight risk among low-income preschool children. Pediatrics 2002, 109, 1028-1035. [CrossRef] [PubMed]

16. Jiang, X.X.; Hardy, L.L.; Ding, D.; Baur, L.A.; Shi, H.J. Recreational screen-time among Chinese adolescents: A cross-sectional study. J. Epidemiol. 2014, 24, 397-403. [CrossRef] [PubMed]

17. GSM Association. The Mobile Economy. 2017. Available online: https://www.gsma.com/mobileeconomy (accessed on 28 June 2017).

18. China E-Commerce Research Center. Smartphone Dependence Survey Report. Available online: http: / / news.xinhuanet.com/info/2016-03/25/c_135222476.htm (accessed on 25 March 2016).

19. Leung, L.; Wei, R. More than just talk on the move: Uses and gratifications of the cellular phone. J. Mass Commun. Q. 2000, 77, 308-320. [CrossRef]

20. Crespo, C.J.; Smit, E.; Troiano, R.P.; Bartlett, S.J.; Macera, C.A.; Andersen, R.E. Television watching, energy intake, and obesity in us children: Results from the third national health and nutrition examination survey, 1988-1994. Arch. Pediatr. Adolesc. Med. 2001, 155, 360-365. [CrossRef] [PubMed]

21. Thomée, S.; Härenstam, A.; Hagberg, M. Computer use and stress, sleep disturbances, and symptoms of depression among young adults-A prospective cohort study. BMC Psychiatry 2012, 12, 176. [CrossRef] [PubMed]

22. Liu, A.L.; Cui, Z.H.; Hu, X.-Q. Determinants of television watching among people in China. Chin. J. Prev. Control Chronic Non-Commun. Dis. 2008, 16, 114-116. (In Chinese)

23. Gordon-Larsen, P.; Mcmurray, R.G.; Popkin, B.M. Determinants of adolescent physical activity and inactivity patterns. Pediatrics 2000, 105, e83. [CrossRef] [PubMed]

24. National Bureau of Statistics of the People's of China. China Statistical Yearbook (2016). Available online: http:/ / www.stats.gov.cn/tjsj/ndsj/2016/indexch.htm (accessed on 28 June 2017).

25. Kikuchi, H.; Inoue, S.; Sugiyama, T.; Owen, N.; Oka, K.; Shimomitsu, T. Correlates of prolonged television viewing time in older Japanese men and women. BMC Public Health 2013, 13, 213. [CrossRef] [PubMed]

26. USC Bureau. Computer and Internet Use in the United States. Available online: http://www.mendeley. com/research/computer-internet-united-states-5/44 (accessed on May 2013).

27. Flores-Mir, C.; Palmer, N.G.; Northcott, H.C.; Huston, C.; Major, P.W. Computer and internet usage by Canadian dentists. J. Can. Dent. Assoc. 2006, 72, 145. [PubMed] 
28. Wang, L.; Xu, X.; Baker, P.; Chao, T.; Lei, Z.; Qi, H.; Yong, Z. Patterns and associated factors of caesarean delivery intention among expectant mothers in China: Implications from the implementation of China's new national two-child policy. Int. J. Environ. Res. Public Health 2016, 13, 686. [CrossRef] [PubMed]

29. Xu, X.; Liu, S.; Rao, Y.; Shi, Z.; Wang, L.L.; Sharma, M.; Zhao, Y. Prevalence and sociodemographic and lifestyle determinants of anemia during pregnancy: A cross-sectional study of pregnant women in China. Int. J. Environ. Res. Public Health 2016, 13, 908. [CrossRef] [PubMed]

30. Yang, Z.; Wang, J.; Li, C.; Lu, M.; Karen, E.; Zhang, J.; Quan, H. Comparing public and private hospitals in China: Evidence from Guangdong. BMC Health Serv. Res. 2010, 10, 76.

31. Bowman, S.A. Television-viewing characteristics of adults: Correlations to eating practices and overweight and health status. Prev. Chronic Dis. 2006, 3, A38. [PubMed]

32. Al-Qahtani, K. Mobile phone use and the risk of parotid gland tumors: A retrospective case-control study. Gulf J. Oncol. 2016, 1, 71-78.

33. State Council of the PRC (2016) Outline of “Healthy China 2030" Program. Available online: http:/ / news. xinhuanet.com/health/2016-10/25/c_1119786029.htm (accessed on 25 October 2016).

34. Davison, K.K.; Edmunds, L.S.; Wyker, B.A.; Young, L.M.; Sarfoh, V.S.; Sekhobo, J.P. Feasibility of increasing childhood outdoor play and decreasing television viewing through a family-based intervention in WIC, New York state, 2007-2008. Prev. Chronic Dis. 2011, 8, A54. [PubMed]

35. Ren, L. "Watch TV" has become "play mobile phone". Vis. Field 2015, 2, 19. (In Chinese)

36. Gao, L.L.; Larsson, M.; Luo, S.Y. Internet use by Chinese women seeking pregnancy-related information. Midwifery 2013, 29, 730-735. [CrossRef] [PubMed]

37. Li, M.; Liu, S. Psychological status of first pregnant women and second child pregnant women. Chin. Med. Equip. 2017, 14, 8. (In Chinese)

38. ZOL (Zhong Guan Cun Zai Xian). Investigation Report of Family Computer Usage in China in 2013. Available online: http://news.xinhuanet.com/info/2013-05/22/c_132401033.htm (accessed on 28 June 2017).

39. Xu, X.; Liu, D.; Zhang, Z.; Sharma, M.; Zhao, Y. Sleep duration and quality in pregnant women: A cross-sectional survey in China. Int. J. Environ. Res. Public Health 2017, 14, 817. [CrossRef] [PubMed]

40. Davis, A.M.; Wambach, K.A.; Nelson, E.L.; Odar, C.; Lillis, T.; Mckinley, A.; Gallagher, M. Health behavior change in pregnant women: A two-phase study. Telemed. J. E Health 2014, 20, 1165-1169. [CrossRef] [PubMed]

41. Padmapriya, N.; Shen, L.; Soh, S.E.; Shen, Z.; Kwek, K.; Godfrey, K.M.; Gluckman, P.D.; Chong, Y.S.; Saw, S.M.; Müller-Riemenschneider, F. Physical activity and sedentary behavior patterns before and during pregnancy in a multi-ethnic sample of Asian women in Singapore. Matern Child Health J 2015, 19, 2523-2535. [CrossRef] [PubMed]

42. Ma, G.; Kong, L. Investigation Report on Nutrition and Health of Chinese Residents. Nine. 2002 Behavior and Lifestyle; People's Medical Publishing House: Beijing, China, 2006.

43. Chen, X.R.; Jiang, Y.; Wang, L.M.; Li, Y.C.; Zhang, M.; Hu, N.; Zhao, W.H. Leisure-time physical activity and sedentary behaviors among Chinese adults in 2010. Chin. J. Prev. Med. 2012, 46, 399-403. (In Chinese) [CrossRef]

(C) 2018 by the authors. Licensee MDPI, Basel, Switzerland. This article is an open access article distributed under the terms and conditions of the Creative Commons Attribution (CC BY) license (http://creativecommons.org/licenses/by/4.0/). 